Reinhard Fiehler

\title{
Über zwei Probleme bei der Untersuchung gesprochener Sprache
}

Die wissenschaftliche Beschäftigung mit gesprochener Sprache wird m.E. durch zwei grundlegende Probleme behindert, die ich in diesem Beitrag verdeutlichen möchte.

Das erste Problem bestcht darin, daß der erkenntnismäßige Zugang zu Phänomenen und Eigenschaften gesprochener Sprache durch ein, written language bias (Linell 1982), dem sowohl das alltagsweltliche Sprachbewußtsein wic auch dic sprachwissenschaftliche Reflexion über Sprache unterliegen, systematisch erschwert und verstellt wird.

Das zweite Problem betrifft dic Frage, ob das, was als gesprochene Sprache bezeichnet wird, eigentlich hinreichend homogen ist, um als einheitlicher Untersuchungsgegenstand gelten zu können, über den generell geltende Aussagen möglich sind.

\section{Das written language bias und seine Folgen für die Analyse gesprochener Sprache}

\subsection{Die Ursachen des written language bias}

Auch wenn die Begriffe, gesprochene Sprache' und, geschriebene Sprache‘ häufig als Paar auftreten und so als Untersuchungsgegenstände gleichen Rangs erscheinen, ist doch der erkenntnismäßige Zugang zu ihnen nicht gleichartig. Es führt kcin direkter Weg zur gesprochenen Sprache, sondern ihre Erkenntnis erfolgt in weiten Bereichen vermittelt über das, was wir von geschriebener Sprache wissen.

Unter den Bedingungen einer entwickelten Schriftlichkeit ist das gesellschaftliche Sprachbewußtscin schriftsprachlich geprägt. Unsere Vorstellungen darüber, was Sprache ist, leiten sich primär aus dem Umgang mit und der Reflexion von geschricbener Sprache her. Dic Gründe, warum die geschricbene und nicht die gesprochene Sprache das Sprachbewußtscin prägt, sind vielfältig. Ich will nur drei kurz ins Gedächtnis rufen:

- Die Schwicrigkeiten der Textproduktion richten das Bewußtsein stark auf dic Strukturen und Eigenschaften der geschriebenen Sprache. Dic Leichtigkeit und der automatische Charakter des Sprechens hingegen bewirken, daß gesprochene Sprache nicht in gleicher Weise ins Zentrum der Aufmerksamkeit und des Sprachbewußtseins rückt.

- Die,Anschaulichkeit' und die Dauerhaftigkeit von Texten - im Gegensatz zur Auditivität und Flüchtigkeit der gesprochenen Sprache - begründen ihre objektmäßige Präsenz und haben seit jeher die Reflexion schriftlicher Texte systematisch begünstigt.

- Geschriebene Sprache wird gesellschaftlich als wichtiger angesehen und höher bewertet als gesprochene. Entsprechend groß ist der Aufwand, der für den Schriftspracherwerb und die Schulung der Schreibfähigkeiten getrieben wird. So 
besitzt in der schulischen Sozialisation die Schriftsprache eindeutig das Primat. Schriftspracherwerb und das Erstellen aller Formen schriftlicher Texte haben dort ein deutliches Übergewicht gegenüber der Schulung mündlicher Kommunikation.

Dies und weiteres tragen dazu bei, daß das Bild von Sprache durch die Schriftsprache bestimmt wird. Die geschriebene Sprache prägt aber nicht nur das gesellschaftliche Sprachbewußtsein, dies gilt gleichermaßen auch für die Sprachwissenschaft als Ort der systematischen Reflexion von (Schrift-)Sprache. Das written language bias betrifft dort auf der einen Seite den Untersuchungsgegenstand und auf der anderen die Kategorien zur Analyse von Sprache.

\subsection{Untersuchungsgegenstand}

Zentraler Untersuchungsgegenstand der Sprachwissenschaft waren in ihrer Geschichte bisher (außer im Zusammenhang mit der Lautlichkeit der Sprache) de facto $^{1}$ schriftliche Texte oder Beispielssätze, die auf der Grundlage cines schriftsprachlich geprägten Bewußtseins schriftnah produziert werden. Nur sie waren (als Texte) dauerhaft gegeben und so einer wiederholten Betrachtung und detaillierten Analyse zugänglich. Wissenschaftliche Sprachreflexion war und ist also - allein schon aus Gründen der Verfügbarkeit des Gegenstandes - weitgehend Reflexion von Texten und damit von Schriftlichkeit.

„Die mangelnde Beachtung der Verschiedenartigkeit von GSCHS und GSPS in der sprachtheoretischen Erörterung indes war in der sprachwissenschaftlichen Praxis Voraussetzung für cine naive Gleichsetzung der Sprache schlechthin mit der GSCHS. Wic selbstverständlich wurden Sprachuntersuchungen auf der Grundlage ausschlicßlich von geschricbenen Äußerungen (Texten) vorgenommen, zumal dic Dokumentation mündlicher Rede damals technisch kaum möglich war. Letztlich sind Sprachuntersuchungen aus dieser Zeit Untersuchungen von GSCHS." (Ludwig 1980, 324)

Anders als die geschriebene Sprache ist die gesprochene ein flüchtiger Gegenstand, was seine Untersuchbarkeit einschränkt und seine Untersuchung in besonderer Weise schwierig gestaltet: Entweder ist man auf die Erinnerung angewiesen, oder aber es bedarf technischer Möglichkeiten der Konservierung von Äußerungen und Gesprächen.

' Im Widerspruch zu ihrer Analysepraxis hat in der Geschichte der Sprachwissenschaft die Auffassung, daß die gesprochene Sprache gegenüber der geschricbenen das Primat besitzt, eine wichtige Rolle gespiclt:

"In der genannten Periode [ 20. Jahrhunderts"] dominiert in der Sprachwissenschaft die Vorstellung vom absoluten Primat (man könnte sagen von ,der linguistischen Legitimität') der gesprochenen Sprache und des akustischen Charakters gesprochener Âußerungen." (Vachek 1976, 241)

In drastischer Deutlichkeit findet sich diese Position nicht nur bei den Junggrammatikern, sondern auch bei de Saussure:

„Sprache und Schrift sind zwei verschiedene Systeme von Zeichen; das letztere bestcht nur zu $\mathrm{dem} Z$ weck, um das erstere darzustellen. Nicht die Verknüpfung von geschriebenem und gesproche$\mathrm{ncm}$ Wort ist Gegenstand der Sprachwissenschaft, sondern nur das letztere, das gesprochene Wort allein ist ihr Objekt." (de Saussure ${ }^{2} 1967,28$ )

Dieses Primat ist als historisches, strukturelles, funktionales und biologisches formuliert worden (vgl. Lyons 1983, 19-25). 
Die Entwicklung und Verbreitung entsprechender technischer Geräte zur Konservierung und Reproduktion von Gesprächen und Interaktionen (Plattenspieler, Tonbandgeräte, Kassettenrekorder, Videokameras) ist so eine wesentliche Voraussetzung für eine detaillierte wissenschaftliche Untersuchung von mündlicher Kommunikation. Setzt man cine bestimmte Ausgerciftheit und Verbreitung solcher Geräte voraus, kann man sagen, daß sie erst seit den 60 er Jahren des 20 . Jahrhunderts gegeben ist.

Eine zweite wesentliche Voraussetzung ist dic Entwicklung von Verfahren zur Verschriftlichung (Transkription) konservierter Gespräche. Transkriptionen ermöglichen eine Vergegenwärtigung und ,Betrachtung' der Äußerungen und Gespräche, wie sic allein durch das Abhören der Aufzeichnung nicht zu erreichen ist. Die Entwicklung solcher Transkriptionssysteme für sprachwissenschaftliche Zwecke (vgl. für einen Überblick z.B. Ehlich/Switalla 1976) erfolgte Hand in Hand mit dem Einsatz der genannten Gerätc. Erst durch das Zusammenspicl von reproduzierbaren Aufnahmen und Transkriptionen wird gesprochene Sprache in einem hinreichenden Detaillierungsgrad untersuchbar, und erst von diesem Zeitpunkt an kann sie überhaupt zu einem ernsthaften und gleichwertigen Untersuchungsgegenstand der Sprachwissenschaft werden.

\subsection{Analyse- und Beschreibungskategorien}

Dic Schrift- und Textlastigkeit der Sprachwissenschaft betrifft aber nicht nur den Untersuchungsgegenstand, sondęrn auch dic Analyse- und Beschreibungskatcgorien als Untersuchungsinstrumente. Die überwiegende Zahl der linguistischen Kategorien wurde in der und für die Analyse geschriebener Texte entwickelt und dann in Grammatiken zu einem relativ festen Satz von Analyse- und Beschreibungskategorien kanonisicrt. Beispicle für solche Kategorien sind ,Satz', Wort', ,Anakoluth', ,Elision' etc. Diese grammatischen Beschreibungskategorien sind - wic alle Kategorien - funktional ihrem Gegenstand angepaßt, und das heißt der Analyse und Beschreibung von geschriebener Sprache. Diese schriftsprachlich orientierten Analyse- und Beschreibungskategorien sind zudem das einzige voll entwickelte Kategoriensystem. Ein Kategoriensystem, das in ähnlicher Weise funktional auf die gesprochene Sprache zugeschnitten wäre, existiert im Moment nur in Ansätzen.

So sind das schriftsprachlich dominierte Sprachbewußtsein und dic für dic Schriftsprache entwickelten Analysekategorien zwangsläufig die Grundlage für das Verständnis und dic Erkenntnis von gesprochener Sprache: Gesprochene Sprache wird durch dic Brille der geschriebenen wahrgenommen, sic ist das Modell für das Vcrständnis von Mündlichkeit.

Günther (1995) hat diese These (im Anschluß an Olson 1993) auch in einer historischen Perspektive ausbuchstabiert: Er stellt dar, daß die Schriftsprache nicht statisch ist, sondern sich in einem geschichtlichen Prozeß entwickelt, in dem er eine scmantische, eine phonographische und eine grammatische Phase unterscheidet. Für alle drei Phasen exemplifiziert er seine zentrale These: „Die Schrift fungiert als Modell für die (Analyse der) Lautsprache." (Günther 1995, 17). So zeigt cr für die phonographische Phase, wie die Segmentierung der gesprochenen Sprache in Wörter an die räumlich-diskrete Organisation der visuellen Symbole gebunden ist:

„Das andere Medium ist es, das überhaupt erst, modellhaft, die Vorstellung diskreter Einheiten im kontinuierlichen Lautstrom ermöglicht. Wo visuell im Raum eine Einheit begreifbar wird, kann man auch auditiv im Zeitverlauf nach Abschnitten suchen. Den 
visuellen Einheiten wird in der Lautsprache etwas zugeordnet: Die Schreiber entdecken, daß man entsprechende Einheiten auch in der Lautsprache rekonstruieren kann. Sie sehen zum ersten Mal gesprochene Wörter." (Günther 1995, 19)

Die Entwicklung der Schreibung in der grammatischen Phase (vgl. die instruktive schematische Darstellung in Günther 1995, 21) ist dadurch gekennzeichnet, daß zunehmend grammatische Kategorien, die der Analyse geschriebener Sprache dienen, auch im Schriftbild visualisiert werden:

„Im Geschriebenen wird die grammatische Organisation sichtbar." (Günther 1995, 22)

Damit sind zentrale Kategorien zur Analyse von Schriftlichkeit nicht abstrakt oder äußerlich, sondern sie manifestieren sich in der Form der Schriftlichkeit. Sic sind dort vergegenständlicht und jeder Blick auf einen Text führt sie vor Augen. So wird das ,Wort' (was schriftsprachgeschichtlich keineswegs immer so war) durch dic Spatien sichtbar, der ,Satz' durch die Großschreibung am Anfang und den abschließenden Punkt, der ,Nebensatz' durch das Komma, das ,Hauptwort' durch seine Großschrcibung (zumindest in der deutschen Schriftsprache) etc. Diesc Katcgorien werden im Entwicklungsprozeß der Schriftsprache als (sich verändernde) Form der Schriftlichkeit ausgearbeitet. Einmal entwickelt, ist die Aktivierung und Anwendung dieser Kategorien Voraussetzung jeder korrekten Textproduktion. Nicht zuletzt auch dieses Faktum macht deutlich, wie permanent und intensiv sic prägenden Charakter für das Sprachbewußtsein haben.

Sind diese Kategorien am Schriftlichen entwickelt (und in der Form des Schriftlichen ,festgeschrieben'), wird es auch möglich, sie auf das Mündliche zu übertragen: "Jede Neuentwicklung der Schrift führt zur ,Entdeckung' weiterer Eigenschaften in der Lautsprache" (Günther 1995, 19).

Die stufenweise Entwicklung der Schriftlichkeit ist so die Voraussetzung für die sukzessive Modellierung der gesprochenen Sprache - cben nach dem Modell der Schriftsprache. Ohne sie wäre ein differenziertes Bild der gesprochenen Sprache nicht möglich.

Die Übertragung der Kategorien auf die gesprochene Sprache führt aber auch zu der Erfahrung, daß sie Mündlichkeit nicht voll erfassen. Diese Differenzen, dic sich aus dem Eigencharakter des Mündlichen ergeben und die Spezifika der gesprochenen Sprache betreffen, werden dann aber zunächst nicht mit gegenstandsangemessenen Kategorien belegt, sondern als Abweichungen von den in der geschricbenen Sprache vorgefundenen Verhältnissen beschrieben und kategorial gefaßt: z.B. Elision, Verschleifung, Ellipse, größere Häufigkeit von Anakoluthen in der gesprochenen Sprache etc.

Darüber hinaus werden diese Abweichungen häufig nicht nur konstaticrt, sondern zugleich implizit oder explizit negativ bewertet. Dic Wahrnehmung dieser Abweichungen führt zu Auffassungen, daß gesprochene Sprache häufig fehlerhaft, weniger regelhaft oder chaotisch sei:

"Die geschriebene Sprache tritt als Zensor der mündlichen auf und erteilt ihr das Verdikt, sie sei unrein, unzureichend, negativ zu bewerten." (Ehlich 1986, 77-78)

Einen anderen Ausweg aus diesem Problem der Wahrnehmung von Abweichungen stellt der (normative) Versuch dar, Mündlichkeit an das Modell der Schriftlichkeit anzupassen. Seinen prominentesten Ausdruck findet dieses Programm in der (pädagogischen) Maxime ,Sprich im ganzen Satz' oder in der Wertschätzung des v/ir-cedruirkt-Redens 
Wenn gesprochene Sprache nach dem Modell und durch die Brille der geschricbenen erkannt wird, dann heißt das, daß auch Vorstellungen über die Art der Einheiten und über Wohlgeformtheitsbedingungen für Einheiten aus dem Bereich der geschriebenen auf die gesprochene Sprache übertragen werden. Was die Einheiten angeht, sind es vor allem die Kategorien, Satz', Wort' und ,Buchstabe', die hier relevant werden. ${ }^{2}$ Buchstabe, Wort und Satz prägen dem Gesprochenen eine Segmenticrung nach ihrem Vorbild auf. Auf der Ebene von Buchstabe und Wort kommt dabei die Vorstellung diskreter Laute und Wörter zum Tragen, die den Verhältnissen im Mündlichen allerdings wenig gerecht wird. ${ }^{3}$ Die Übertragung der Wohlgeformtheitsvorstellungen für schriftsprachliche Wörter und Sätze führt zu der Feststellung, daß das Mündliche diesen in vieler Hinsicht nicht entspricht und - wie oben schon ausgeführt - zur Ausbildung von Defizienzkategorien.

Darüber hinaus wird aber auch cine Produktperspektive an die gesprochene Sprache herangetragen. Da Texte in der Regel nur als materielle Endprodukte gegeben sind, die den Entstehungsprozeß nicht mehr erkennen lassen, wird diese Sichtweise auch für gesprochene Sprache konstitutiv. Ich werde darauf zurückkommen, daß dies nicht gegenstandsangemessen ist.

Das Verständnis der gesprochenen Sprache nach dem Modell der geschriebenen und dic Übertragung von Analyse- und Beschreibungskategorien, dic für das Schriftsprachliche entwickelt wurden, in den Bereich der Mündlichkeit erscheint so lange nicht problematisch, wie man annimmt, daß beides in einheitlicher Weise Sprache ist und daß die Unterschiede eher akzidentiell als essentiell sind. ${ }^{+}$Solche Positionen finden sich in großer Zahl in der linguistischen Literatur. Nur zwei Zitate als Beleg:

"Als Fazit bleibt für mich deshalb: Eigene Sprachvarietäten: „gesprochene Sprache“ vs. "geschriebene Spracbe" gibt es nicht, wenn man als Kriterium, wic bei den anderen Varictäten den langue-Begriff benutzt und nach jewcils eigenen, nicht im anderen Medium zulässigen Strukturmitteln und Relationen fragt." (Steger 1987, 57)

"Insbesondere syntaktische Strukturen sind offensichtlich unabhängig von den spezifischen Eigenschaften der lautlichen oder graphischen Realisierung. Es scheint keine Beispiele dafür zu geben, daß eine syntaktische Konstruktion in der geschriebenen Sprache korrekt, in der gesprochenen dagegen inkorrekt ist oder umgekehrt, d.h., in dieser Hinsicht gilt eine cinheitliche hochsprachliche Norm." (Motsch 1992, 246)

An der Einhcitlichkcit zu zweifeln, besteht zunächst auch kein Grund: Beides dient der Verständigung und weist dem Anschein nach vicle Ähnlichkeiten aus, zumal durch die Brille der geschriebenen Sprache dominant nur dic Phänomene und Berei-

\footnotetext{
2 Schriftsprachliche Einheiten wie ,Absatz', ,Kapitel' oder ,Text' bleiben dabei irrelevant, weil ihnen eine dirckte Entsprechung im mündlichen Bcreich fehlt.

${ }^{3}$ "So wundert es nicht, daß manche Sprachwissenschaftler die Phonemstruktur für cin Epiphänomen des Alphabets halten und eine Projektion von Schriftförmigem auf den kontinuierlichen Lautfluß vermuten." (Krämer 1996, 104)

+ Eine solche Position ist konstitutiv für Sprachkonzeptionen, die Sprache als medienunabhängiges bzw. -ncutralcs Phänomen verstehen: „Man muß zwischen den sprachlichen Signalen und $\mathrm{dem}$ Medium, in dem die Signale realisiert werden, unterscheiden. [...] Soweit Sprache in diesem Sinne unabhängig von dem Medium ist, in dem die sprachlichen Signale realisiert werden, behaupten wir, daß Sprache dic Eigenschaft hat, nicht an cin Medium gebunden zu scin." (Lyons 1983, 19-20). Solche Konzeptionen sind in der jüngeren Geschichte der Sprachwissenschaft die gängigen. Zu ihnen gehören insbesondere auch Auffassungen, die cin medienneutrales Sprachsystem postulieren.
} 
che wahrgenommen werden, wo Entsprechungen bestehen oder wo sie zumindest hineinprojiziert werden können.

Problematisch ist eine Übertragung von Kategorien jedoch, wenn diese Einheitlichkeit nicht der Sache nach besteht, sondern lediglich ein Resultat der Projektion ist. In der Tat ist die Einheitlichkeit bzw. Strukturäquivalenz von gesprochener und geschriebener Sprache in verschiedener Hinsicht und mit unterschiedlichen Argumenten bestritten worden. So vertritt z.B. Krämer $(1996,103)$ die These, daß es begründete $Z$ weifel daran gibt, daß die mündliche Sprache ein disjunktives und endlich differenzierbares Symbolschema ${ }^{5}$ darstellt, wie es bei der geschriebenen der Fall ist.

"Doch ist zu vermuten, daß der vielleicht folgenreichste Aspekt, der mit dem vorliegenden Schriftbegriff zu gewinnen ist, gerade darin besteht, daß er eine medienzentrierte Abgrenzung zwischen Sprachen und Schriften, damit auch zwischen geschriebener und gesprochener Sprache erlaubt. Und diese liegt nicht in der Unterscheidung von Phonischem und Graphischem, von Sprache der Nähe und der Distanz, sondern darin, daß mündliche Sprachen nicht als disjunktive, endlich differenzierbare Schemata gelten können." (Krämer 1996, 103)

Auf diesem Hintergrund kommt sie zu der These:

„Der sprachwissenschaftliche „Skriptizismus“ besteht darin, Sprache zu behandeln, als ob sie ein disjunktives, endlich differenzierbares System sei. Dies ist eine für das Studium der Sprache unabdingbare wissenschaftliche Fiktion." (Krämer 1996, 107; gemeint ist mit ,Sprache' hier gesprochene Sprache; R.F.)

Eine skeptische Haltung, was die Übertragbarkeit grammatischer Kategorien angeht, findet sich aber auch schon in der Frühphase der Entwicklung der Gesprochenen-Sprache-Forschung in der Bundesrepublik:

„Im hier behandelten Zeitraum [1965-1973; R.F.] kommt es dabei zu cincr Kontroverse, ob die gesprochene Sprache überhaupt mit den aus der Schriftsprache gewonnenen grammatischen Kategorien beschrieben werden kann und nicht vielmehr andersartige Beschreibungsmodelle erfordere." (Betten 1977, 346)

Die Erkenntnis gesprochener Sprache wird also erschwert und die Dominanz der geschriebenen Sprache im Sprachbewußtsein immer von Neuem perpetuicrt durch die Tatsache, daß zentrale Analyse- und Beschreibungskategorien schriftsprachlich ausgerichtet sind. Es bedarf deshalb der theoretischen Reflexion und Kritik der gängigen Analyse- und Beschreibungskategorien sowie der Entwicklung modifizierter, gegenstandsangepaßter Kategorien, um die Ablösung von der Schriftorientierung nicht nur auf der Daten-, sondern auch auf der Kategorienebene vollziehen zu können. Während bei der Verfügbarkeit gesprochener Sprache durch die Entwicklung von Aufzeichnungs- und Wiedergabegeräten sowie von Transkriptionssystemen qualitative Fortschritte erzielt wurden, hat der Prozeß der Reflexion und Entwicklung von Kategorien bisher keinen vergleichbaren Stand erreicht.

${ }^{5}$ Die strukturellen Eigenschaften, um die es hier geht, gruppieren sich um die Frage, ob Materialien Zwischenräumlichkeit zulassen oder ob sie dicht sind. “ (Krämer 1996, 99)

"Während die Disjunktivität sicherstellt, daß Marken, die zu demselben Zeichen gehören, fürcinander austauschbar sind, gewährleistet die Differenzierbarkeit, daß es überhaupt möglich ist, korrekt zu entscheiden, daß zwei Marken zu demselben Zeichen gehören.“ (Krämer 1996, 101). Zu genaueren Ausführungen zu Schrift als disjunktivem und endlich differenzierbarem Symbolschema vgl. Krämer (1996, 99ff.). 
Dic Entwicklung gegenstandsangemessencr Analyse- und Beschrcibungskategorien für mündliche Kommunikation verlief dort relativ unproblematisch, wo es um Phänomene geht, die keine unmittelbare Entsprechung im schriftlichen Bereich haben. In dem Maße, wie authentische gesprochensprachliche Daten zur Verfügung standen, setzte zunächst im Rahmen der Pragmatik und dann in den verschiedenen Varianten der Gesprächsforschung der Prozeß der Kategorienentwicklung ein, um die Andersartigkeit dieses Materials zu erfassen. Dabei wurden aus dem Spektrum der Mündlichkeit vor allem die Formen betrachtet, bei denen eine Kopräsenz der Parteien und die Wechselseitigkeit der Wahrnehmung gegeben ist und bei denen kommunikative Aktivitäten zudem die zentrale Tätigkeitsform darstellen. ${ }^{6}$ So waren es vor allem Phänomenc der Interaktivität, für die Kategorien entwickelt wurden. In den Blick genommen wurden zunächst die Gesprächsorganisation (turn-taking) und verschiedene Aspekte der Äußerungsorganisation (Gliederungssignale, Höreräußcrungen, Reparaturen), in der Folge dann kommunikative Verfahren (Präferenzorganisation) und Strukturen von Gesprächen (Muster, Handlungsschemata) sowic spezifische Aufgabenkonturen cinzelner Gesprächstypen (Erzählungen, Beratungen ctc.). Als Resultat dieser Entwicklungen hat sich die Gesprächsforschung als Disziplin mit einem eigenständigen Gegenstandsbereich und ciner spezifischen Methodologic etablicrt.

Gan $z$ anders steht es um dic Kategorienentwicklung im grammatischen Bercich. Da hier ein entwickeltes Kategorieninventar aus dem Bereich des Schriftlichen zur Verfügung steht, wurden diese Kategorien zunächst für die Beschreibung des Mündlichen übernommen und, wenn ihre Übertragung Probleme bereitete, gegebenenfalls adaptiert. Exemplarisch läßt sich dies an der Frage nach den grundlegenden Einheiten des Mündlichen verfolgen. Hier wurde zunächst versucht, eine der zentralen Einheiten des Schriftlichen - den Satz - auf das Mündliche zu übertragen. In dem Maßc, wie dies Schwicrigkeiten bereitete, wurde die Kategoric ,Satz' entsprechend modifiziert bzw. es wurden andere Kategorien (Äußerungscinheit, turn, sprachliche I-landlung, Äußcrung, intonation unit etc.) ins Spicl gebracht (vgl. hicrzu Fichler/Barden/Elstcrmann/Kraft i.V., Abschnitt II.2).

Die gegenwärtige Situation bei der kategorialen Erfassung grammatischer Eigenschaften der gesprochenen Sprache ist gekennzeichnet durch drei Tendenzen: Eine Strategic besteht nach wic vor in der Übernahme und Adaption von Kategorien der traditioncllen Grammatik. Der zweite Zugang besteht in ciner handlungs- und funktionsorienticrten Reinterpretation traditioneller Katcgorien. Dicse Vcränderung der Perspektive ist in der Regel auch mit einer Veränderung der Kategoriennamen verbunden. Eine dritte Strategie versucht, grammatische Phänomene aus den Grundbedingungen gesprochener Sprache, insbesondere aus den Bedingungen der Intcraktivität, herzuleiten und kategorial zu fassen. Dies führt häufig zu einer Erwciterung des Kategorieninventars für die Analyse gesprochener Sprache. Diesc verschiedenen Zugänge sind im Einzelfall jedoch häufig nicht klar voneinander abzugrenzen.

\footnotetext{
${ }^{6}$ Dies ist keine Selbstverständlichkeit, wenn man sich die Vielzahl kommunikativer Praktiken vor Augen stellt, bei denen dies nicht der Fall ist. Zu denken ist z.B. an mündliche Praktiken wie die kommunikative Organisation von Arbeitsprozessen, die Beichte, das Besprechen eincs Anrufbeantworter, das Verlesen von Nachrichten etc.
} 
(1) Insbesondere die Überzeugung, daß das, was in den Grundzügen gleichartig ist, auch mithilfe der gleichen Kategorien beschrieben werden kann, findet in vielfältigen Versuchen Ausdruck, Kategorien aus dem schriftsprachlichen Bereich zu verwenden bzw. sie so zu modifizieren, daß sie brauchbar werden. Dies betrifft zum Beispiel die Übertragung von Kategorien wie Satz (Kindt 1994, Hoffmann 1996), Anakoluth (Hoffmann 1991), Parenthese (Hoffmann 1998), Ellipse (Selting 1997, Hoffmann 1999) etc. auf den Bereich des Mündlichen. Vor allem für dic Kategoric ,Satz' sind auch entsprechende Adaptionen vorgeschlagen worden, zuletzt die Kategorie des möglichen Satzes bei Selting (1995).

(2) Da traditionelle Kategorien häufig produktorientiert, strukturbezogen oder formbestimmt sind, das Sprechen aber in sehr deutlicher Wcise cin Handlungspro$z e ß$ ist, liegt es nahe, Kategorien in Hinblick auf ihre Funktion im sprachlichen Handeln zu reinterpretieren. So stellt beispielsweise Rehbein (1979) sprachliche Elemente, die der, eigentlichen` Äußerung vor- oder nachgestellt sind und dic traditionell, soweit sic überhaupt kategorial gefaßt werden, als Vokative, Imperative oder tag-questions bezeichnet werden, in den Handlungskontext der Hörersteuerung:

„Meines Erachtens ist dieses Phänomen konsequent in den erörterten Zusammenhang des kommunikativen Apparats der wechselseitigen Steuerung zu stellen, um von dorther erst die syntaktische Analyse zu unternehmen." (Rehbein 1979, 60)

Er leistet damit eine Zusammenfassung und funktionale Reinterpretaion der traditionellen Kategorien. Allerdings wird die Funktionalität dieser Elemente nicht hinreichend auch im Kategoriennamen zum Ausdruck gebracht, wenn Rehbein sie als ,Sprechhandlungsaugmente' oder häufiger noch als, Vorschaltungen' bzw. ,Nachschaltungen' anspricht. Diese Begrifflichkeit ist deutlich strukturell und nicht funktional geprägt. Am Beispiel der Kategorie ,Herausstellung werde ich im Anschluß cine weitere Reinterpretation vorstellen.

Was traditionelle, strukturbezogene Kategorien bezeichnen, wird bei dieser Strategic also rekonstruiert in Hinblick auf seine Funktion(en) im Prozeß des Sprechens, d.h. auch in Hinblick auf seine Charakteristik und Qualität als Handlung (funktional-handlungsmäßige Reinterpretation von Kategorien). Reinterpretation bedcutet, daß die kommunikative Funktion von sprachlichen Mitteln oder Strukturen expliziert und dann in einem ,sprechenden' Kategoriennamen kondensiert wird. Im beschricbenen Sinn reinterpretierte Kategorien sind damit prozeßorientiert, es sind I landlungs- bzw. Aktivitätskategorien.

(3) Die dritte Strategie wird von der Auffassung getragen, daß sprachliche Mittel und Strukturen Werkzeuge sind zur Bearbeitung von rekurrenten Aufgaben, dic sich den Beteiligten im Prozeß der sprachlichen Interaktion stellen. In dem Maße wie diese Aufgaben rekurrent sind, bilden sich in der interaktiven Praxis in einem Prozeß der Grammatikalisierung bestimmte Mittel und Strukturen heraus, dic geeignet sind, diese Aufgaben in effektiver Weise zu realisieren.

"In this interpretation, grammar stands in a relatively intimate relation to social interaction. It is designed for interactional ends and as such must reckon with the architecture and dynamics of turns, sequences, activities, participant frameworks, stances, trouble, expectations, contingencies, and other relevant interactional actualities." (Schegloff/ Ochs/Thompson 1996, 36) 
Bei diesem Ansatz wird nachgezeichnet, wie Grammatik aus den Grundbedingungen mündlicher Kommunikation, insbesondere von Interaktivität, hervorgeht. ${ }^{7}$ So werden z.B. die verschiedenen Formen von Nachträgen, aber auch generell Phänomene der Wortstellung mit der Turnorganisation in Zusammenhang gebracht (Aucr 1991, Uhmann 1993). Gohl/Günthner (1999) und Günthner (1999) konstatieren einen partiellen Kategorienwechsel bei weil und obwobl von unterordnenden Konjunktionen zu Diskursmarkern als Resultat eines Grammatikalisicrungsprozesses. Auer (1998, 301-303) argumentiert für eine entsprechende Grammatikalisierung von Matrixsätzen zu Diskursmarkern.

\subsection{Kritik und Rekonstruktion der Kategorie ,Herausstellungen“}

Im folgenden soll exemplarisch die Kategorie ,Herausstellung' daraufhin überprüft werden, ob sic das betreffende Phänomen der gesprochenen Sprache angemessen crfaßt und im Anschluß daran der Versuch einer Rekonstruktion unternommen werden.

Seit Altmanns Arbeit „Formen der ,Herausstellung“ im Deutschen“ (1981) haben dicse Strukturen gerade bei der Analyse mündlicher Kommunikation eine erhebliche Beachtung gefunden.

„Herausstellungstrukturen sind Ausdrücke, die formal-syntaktisch keine vollständigen Sätze sind [...], die aber zum Teil durchaus satzwertige, wenn auch stark elliptische Strukturen darstellen. Zur Auffüllung der Ellipsen sind die zugeordneten Sätze notwendig. Ebenso wird den Herausstellungsstrukturen erst durch diesen zugeordneten Satz dic semanto-pragmatische Funktion zugewiesen." (Altmann 1981, 46)

Ilcrausstellungsstrukturen sind dadurch auffällig, daß sic die Wohlgeformtheitsbedingungen des schriftsprachlichen Satzes nicht erfüllen, sondern gerade in spezifischer Weise von ihnen abweichen. Diese grundlegende Orienticrung am wohlgeformten Satz, der implizit als Vergleichsgegenstand benutzt wird, ist für die Kategorienbildung konstitutiv. Sic kommt auch darin zum Ausdruck, daß in der oben stehenden Bestimmung zunächst dic Frage thematisiert wird, inwicweit dic herausgestellten Ausdrücke satzwertig sind. Dic Kategoric ,Herausstellung' erfaßt also cine Abweichung - vom wohlgeformten schriftlichen Satz. Diese Abwcichungen werden nun aber nicht - was konsequent wäre - als Fehler (ungrammatischer Satz) gewertet, sondern wegen ihres häufigen Auftretens in der gesprochenen Sprache als eigenständige und - in I linblick auf diesen Bercich - wohlgeformte Struktur ctablicrt.

Dic Grundidec der Kategorie ,Herausstellung' besteht darin, die Genese dieser Strukturen dadurch zu erklären, daß Elemente, die keine vollständigen Sätze sind, aus zugrundeliegenden wohlgeformten Sätzen herausgenommen und vor oder nach dem Bezugssatz positioniert werden. Dies ergibt die sogenannten ,Links-' bzw. ,Rechtsversetzungen'. Dieser Terminologic liegt die Vorstellung eines räumlich gegenwärtigen, vor Augen stehenden schriftlichen Satzes zugrunde. Aus ihm werden Elemente, herausgenommen' und räumlich nach links bzw. rechts versetzt. Dic Genese dieser Strukturen wird also als Manipulation an einem vorliegenden wohlgeformten schriftsprachlichen Satz konzeptualisiert. Hierin ebenso wie in den Kategoriennamen ist die schriftsprachliche Orientierung - bei der Erfassung eines primär

\footnotetext{
'Vgl. z.B. Ford (1993), Ochs/Schegloff/Thompson (1996) oder Aucr (1996).
} 
gesprochensprachlichen grammatischen Phänomens! - mit Händen zu greifen. ${ }^{8}$ Man möchte diese Strukturen nach dem Bild des schriftlichen Satzes verstehen, dies gelingt aber - wie die oben zitiere Beschreibung der Struktur belegt - nur um den Preis eines Rekurses auf Elliptizität. Ferner implizieren diese Kategorien eine strukturelle Äquivalenz der Herausstellungen: Von der Struktur her scheint es kcinen prinzipiellen Unterschied zu machen, ob nach links oder rechts herausgestellt wird.

Faßt man diese Beobachtungen zusammen, so erweisen sich dic Kategorien ,Herausstellung' und ,Links-' bzw. ,Rechtsversetzungen' als strukturell, nicht funktional begründet und als produkt- und nicht prozeßorientiert. Ihre schriftsprachliche Orientierung zeigt sich sowohl in den Kategoriennamen wic auch darin, daß für sie das Konzept des wohlgeformten Satzes grundlegend ist. Letztlich ist die Kategorienbildung ,Herausstellung' Resultat einer impliziten und nicht reflektierten Fixiertheit auf die Normalform des schriftlichen Satzes. Sie belegt cindrucksvoll die Dominanz der schriftsprachlichen Orientierung bei der sprachwissenschaftlichen Kategorienbildung.

Die betrachteten Phänomene erscheinen in einem ganz anderen Licht, wenn man davon ausgeht, daß Sprechen ein prozeßhaftes Handeln ist. Die erste Konsequenz einer solchen Sichtweise ist, daß diese Strukturen nicht als räumliche Gebilde verstanden werden dürfen, sondern in ihrer zeitlichen Abfolge, ihrem Nacheinander betrachtet werden müssen. Der Prozeßcharakter des Sprechens erfordert dies. ${ }^{9}$ Ferner ist danach zu fragen, was die einzelnen Bestandtcilc dieser Strukturen leisten, was Sprecher und Hörer mit ihnen tun. Dies crmöglicht cine funktionale und handlungsbezogene Interpretation. Betrachten wir den Fall von ,Linksversetzung' und ,freiem Thema', so leistet das zuerst produzierte Element eine Referenz bzw. eine Thematisierung: Der Sprecher identifiziert etwas bzw. führt ein Thema ein. ${ }^{10}$ Mit dem folgenden Element wird dann etwas über das Referenzobjekt oder das Thema ausgesagt. Dabei kann, muß aber nicht durch cin deiktisches Element noch einmal auf den Referenz- bzw. Thematisierungsausdruck Bezug genommen werden. Die Trennung von Referenz bzw. Thematisie-

${ }^{8}$ Die schriftsprachliche Orientierung ist bei Altmann auch bei dem scinen Untersuchungen zugrundeliegenden Material zu greifen, handelt es sich doch überwiegend um Wiedergaben gesprochener Sprache in Form schriftlich normalisierter Texte, denen eine Satzstruktur aufgeprägt ist:

"Die weitaus meisten Belege [...] fanden sich in Mitschriften mündlicher Rede in ,cntspannten" Kommunikationssituationen, aber auch in ,bereinigten' Mitschriften, wie den Bottroper Protokollen, den Iireiburger Veröffentlichungen zum gesprochenen Deutsch, dann in Untersuchungen wie der von K. Baumgärtner zur ,Syntax der Umgangssprache von Leipzig' (1959), aber auch in Erzeugnissen der ,schönen Literatur', soweit dort mündliche Sprache, unmittclbarer Dialog möglichst realistisch nachgebildet wird. Die ergiebigsten Fundstellen bildeten allerdings Debatten des Deutschen Bundestags sowie der Landtage, und alltägliche Gespräche im Umgang mit Bekannten, Verwandten und Kollegen. Die passenden Belege wurden meist nur isolicrt notiert, anfänglich ohne genaucre intonatorische Angaben." (Altmann 1981, 74)

$9{ }_{n}$ Die Bewegungs-Analyse verkennt also nicht nur die grundlegenden Unterschicde zwischen den beiden Typen von ,Herausstellung" (was durch die parallelisierende Terminologic - Rechts/Linksversetzung, etc. - noch unterstützt wird), sie ist grundsätzlich nicht in der Lage, die Produktion und Rezeption einschlägiger Phänomene unter Berücksichtigung der Zeitlichkeit von Sprache zu erfassen." (Auer 1991, 140)

${ }^{10}$ Auch Altmann (1981, 48 und 50) benennt die Funktion von Linksversetzungen und Freien Themen als Thematisierung, ohne allerdings daraus weiterreichende Konsequenzen zu ziehen. Scheutz $(1997,44-46)$ charakterisiert Linksversetzungen als „Mittel der Referenzkonstitution“: „Der weitaus größte Teil der Bearbeitung potentieller Verständigungsprobleme gilt jedoch der interaktiven Herstellung und Sicherung von Referenz." (46) 
rung und Aussage ist dabei umso wahrscheinlicher, je komplexer und schwieriger dic Referenz bzw. Thematisierung ist. ${ }^{11}$

Auch im klassischen Satz geschicht nichts anderes: Er leistet eine Referenz, und über das Referenzobjekt wird dann etwas ausgesagt. ,Linksversetzung' und ,freies Thema' unterscheiden sich vom klassischen Satz nur dadurch, daß Referenz-bzw. Thematisierungsakt und Aussage deutlicher als eigenständige Handlungen voneinander abgesetzt sind und als separiert markiert werden. Die kommunikativ zu leistenden Aufgaben werden getrennt und sind damit für den Ilörer leichter unterscheid- und nachvollziehbar. Im Satz sind sie stärker integriert und weniger als cigenständige Handlungen erkennbar.

Als Resultat cincr solchen Reinterpretation scheint es sinnvoll, dic betreffenden Phänomene auch begrifflich anders zu fassen, d.h. cinen anderen Kategoriennamen cinzuführen. Denn es wird hier ersichtlich nichts ,herausgestellt' oder, versetzt', sondern es werden lediglich zwei Handlungen deutlicher voneinander abgesetzt, als es im klassischen Satz der Fall ist. Eine mögliche Bezeichnung wäre ,Referenz-Aussagc-Strukturen' bzw. ,Thematisierung-Aussage-Strukturen'.

Eine weitere Konsequenz der Reinterpretation ist die Einsicht, daß ,Linksversetzung' und ,Rechtsversetzung ' nicht einfach richtungsmäßig unterschiedliche, aber ansonsten äquivalente Operationen sind, sondern daß sic jeweils ganz andere Funktionszusammenhänge betreffen. Während ,Linksversetzungen' - wie dargestellt auf einer Separierung von Referenzakt und Aussage beruhen, sind ,Rechtsversetzungen' (Ausklammerungen, Nachträgc, Reparaturen, appositive und konjunktionale Weiterführungen etc.) - wic Auer (1991) ausführlich darlegt - im Kontext der Expansion von Beiträgen und der Turnorganisation zu verstehen. Dic räumlichstrukturelle Betrachtungsweise führt so zu einer Zusammenführung von Phänomenen, die funktional nichts miteinander zu tun haben. ${ }^{12}$

Auch wenn cine solche Kritik der Kategorie ,Hcrausstellung' in den auf Altmann folgenden Arbeiten, gerade denen gesprächsanalytischer Provenienz, schon zum Teil geleistet wurde (Auer 1991, Selting 1993, 1994, Scheutz 1997), war dies jedoch bisher kein Anlaß, eine andere Kategorienbezeichnung einzuführen: Durchgehend werden dic Phänomenc auch weiterhin als, Herausstellung', ,Links-' oder , Rechtsversetzung' angesprochen:

"Jochen Rehbein hat anläßlich eines Vortrags von mir in Mannheim den Terminus ,Linksversetzung' scharf kritisiert, da dicser den Blick auf die real ablaufenden Sprachproduktionsprozesse verstelle und in ethnozentristischer Weise lediglich dic Linearität unseres Schriftsystems abbilde - ich schließe mich dieser Kritik an; gleichwohl verwende ich diese mittlerweile zum terminologischen Allgemeingut gewordenen Begriffe weiterhin, um zusätzliche Aufblähungen des ohnehin reichlich gefüllten Begriffsarsenals zu vermeiden. Der Verwendungszusammenhang in meiner Analyse wird deutlich machen, wie ich diesc. Konstruktionen verstehe." (Scheutz 1997, 52, Anm. 1)

M.E. ist der Rekurs auf ,terminologisches Allgemeingut' unzureichend: Abgesehen davon, daß dadurch ein Stück begrifflicher Klarheit verschenkt wird, birgt dies auch die Gefahr, daß mit der Bezeichnung die implizite Satz- und Produktorientierung sicherlich nicht beabsichtigt, aber unter der Hand - tradiert wird. In jedem Fall ver-

${ }^{11}$ Scheutz $(1997,29)$ gibt Beispiele, die die häufig auftretenden Schwierigkeiten bei der Referenz sehr schön verdeutlichen: z.B. aber der der doktor wólf oder wie der héisst der álte, (.) das mußáuch a ganz a príma kérl sein.

${ }^{12}$ Gleiches gilt z.B. auch für die unter die Kategorie ,A pokoinu' subsumierten Phänomene. 
weist die Tatsache, daß es bisher keine Vorschläge für eine neue Begrifflichkeit gibt, auf das Beharrungsvermögen eingeführter und verbreiteter Kategorien; ferner vor allem aber auch darauf, daß die schriftsprachliche Orientierung des Sprachbewußtseins nicht schon mit ihrer Erkenntnis überwunden ist.

Durch eine solche, hier in Ansätzen vorgeführte Reinterpretation, die prozeßund handlungsorientiert die kommunikative Funktion von sprachlichen Mitteln oder Strukturen rekonstruiert und expliziert, werden die betreffenden Phänomene deutlich gegenstandsadäquater konzeptualisiert. ${ }^{13}$ Auch für weitere grammatische Kategorien ist eine entsprechende Reinterpretation erforderlich. Vorrangig geht es dabei um ein Äquivalent für die schriftsprachlich orientierte Kategorie ,Satz' in der gesprochenen Sprache. Weitere Kategorien, die der Reinterpretation bedürfen, sind ferner z.B. die eng mit dem Satzbegriff zusammenhängenden Kategorien wic ,Ellipse', ,Anakoluth'14, ,Nebensatz' etc.

\section{Die Inhomogenität des Untersuchungsgegenstandes}

Die Unterscheidung und Gegenüberstellung von ,gesprochener Sprache' und ,geschriebener Sprache ist in der Sprachwissenschaft eine ganz geläufige. ${ }^{15} \mathrm{Wcnn}$ nicht generell und allgemein, Sprache' untersucht wird, ist dies eine der ersten und fundamentalen Differenzierungen, mit der Erscheinungsformen der Sprache unterscheiden werden. Ihre Unterscheidung war in den letzten Jahrzehnten Ausgangspunkt für vielfältige theoretische Bemühungen um diese Differenz wie auch für zahlreiche Versuche, mal mehr, mal weniger empirisch gestützt, Unterschicde zwischen ihnen herauszuarbeiten.

Gerade im Kontext des Vergleichs scheint nun diese Gegenüberstellung zu suggerieren, daß es sich hierbei um gleichrangige und jeweils relativ homogene Formen der Verständigung handelt. Im folgenden Abschnitt möchte ich diese Homogenitätsannahme bzw. -unterstellung problematisieren und zeigen, daß dic Konstitution des Gegenstandsbereichs, gesprochene Sprache das Resultat von drei Prozessen ist: der Prototypisierung, der Homogenisierung und der Abstraktion von der Praktikengebundenheit des Sprechens. Im Anschluß daran werde ich in Abschnitt 2.2 das Konzept der kommunikativen Praktiken skizzieren, das den Bereich mündlicher Kommunikation nicht homogenisiert, sondern differenziert.

${ }^{13}$ Für eine vergleichbare Rekonstruktion dieser Strukturen s. Ehlich (1986). Ohne auf die Kategorie ,Herausstellung' Bezug zu nehmen - er thematisiert diese Konstruktion als speziellen Solözismus -, rekonstruiert Ehlich die hörerbezogene Funktionalität der Konstruktion: ${ }_{n}$ Es erweist sich also, daß der Solözismus - funktional ist. [...] Die Beurteilung als Solözismus spricht über den Satz ein Verdikt aus. Die Bestimmung als, pragmatisch-funktional angemessen' tut dies keineswegs - im Gegenteil - die Bewertung ist explizit positiv. Fragen wir nach dem Kriterien, die dem Verdikt zugrunde liegen, so sehen wir: sie beziehen sich offenbar auf bestimmte ,Normalformen' syntaktischer Struktur." (Ehlich 1986, 83-84)

${ }^{14}$ Für eine Reinterpretation dieser Kategorie vgl. Schröder (1998).

${ }^{15}$ Terminologisch gibt es viele Varianten, die auf diese Unterscheidung zielen: Mündlichkeit und Schriftlichkeit, gesprochensprachliche und geschriebensprachliche Kommunikation, mündliche und schriftliche Verständigung, Diskurs und Text, Rede und Schrift etc. Alltagsweltlich ist sie als Unterscheidung von Sprechen und Schreiben präsent. 


\subsection{Die Konstruktion von Einheitlichkeit}

Das Konzept ,gesprochene Sprache' hat seinen Ursprung im Kontext des Vergleichs, und meistenteils wird es in Zusammenhängen relevant, in denen es um dic Gegenüberstellung und Abgrenzung von gesprochener und geschricbener Sprache geht. ${ }^{16}$ In diesen Kontexten liegt das Augenmerk weniger auf der internen Vielfalt gesprochener Sprache als vielmehr auf ihrer Einheitlichkeit. Gesprochene und geschriebene Sprache stellen aber - medial betrachtet - keine homogenen Gegenstandsbereiche dar, sondern sie umfassen jeweils eine Vielzahl sehr unterschiedlicher kummunikativer Praktiken: Sie reichen auf der einen Seite vom Scherzen am Mittagstisch über den Gottesdienst bis hin zum Besprechen eines Anrufbeantworters und auf der anderen Seite vom Notizzettel über dic Bedienungsanleitung für den Computer bis hin zur philosophischen Abhandlung. Was diese Praktiken bei aller Unterschiedlichkeit vereint, ist, daß sie einerseits mündlich und andererseits schriftlich ausgeführt werden. Dic Einheitlichkeit gesprochener Sprache ist also nicht primär in der Sache begründet, sondern sie wird durch verschiedene Abstraktions- und Konstruktionsprozesse hergestellt.

Einen ersten Beitrag zur Vereinheitlichung leistet die Prototypisierung: Sowohl dic mündlichen wic die schriftlichen Praktiken werden untereinander nicht als glcichwertig und -rangig wahrgenommen. Manche dieser Praktiken scheinen uns bessere, genuinere Fälle von Mündlichkeit bzw. Schriftlichkcit zu scin als andere. Dic Vielfalt von kommunikativen Praktiken ist überlagert von Vorstellungen über ihre Prototypik. So erscheint sicherlich vielen das Gespräch von Angesicht zu Angesicht eine typischere Form der gesprochenen Sprache als eine Rede oder ein Telefongespräch und beides immerhin noch typischer als das Rezitieren eines Gedichts. Weniger einheitlich dürften die Auffassungen darüber sein, ob eine Konversation am Tisch oder ein Arzt-Patienten-Gespräch (als Form institutioneller Kommunikation) der typischere Fall von gesprochener Sprache ist. Selbstredend sind auch dicse Vorstellungen über Prototypik nicht homogen, sondern können von Person zu Person sowic im Laufe der Zeit variieren. Bei der Bewertung, daß etwas prototypischer mündlich ist als etwas anderes, spielen u.a. folgende Aspekte cine Rolle:

- dic relative Häufigkeit einer kommunikativen P'raktik im Kommunikationshaushalt ciner Gesellschaft,

- dic Häufigkcit ciner Praktik im individuellen Kommunikationshaushalt und ihre subjektive Bedeutsamkeit,

- Vorstellungen über ihre (historische) Ursprünglichkeit und

- Vorstellungen über ihre Elementarität bzw. ,Reinheit ${ }^{17}$.

Problem diescr hierarchischen Anordnung von Praktiken im Bewußtsein ist, daß sic dic Tendenz fördert, Mündlichkeit bzw. Schriftlichkeit mit der typischsten Praktik bzw. wenigen besonders typischen Praktiken zu identifizieren. Weniger typische

${ }^{16}$ So auch schon bei Behaghel in seinem berühmten Festvortrag "Geschriebenes Deutsch und gesprochenes Deutsch“ (Behaghel 1899), den er vor etwas mehr als 100 Jahren - am 1. Oktober 1899 - auf der Hauptversammlung des Deutschen Sprachvereins hielt.

${ }^{17}$ Dic letzten beiden Aspekte hat Quasthoff im Auge, wenn sie schreibt: „Aus meiner Sicht lassen sich die wesentlichen Bestimmungsstücke mündlicher Kommunikation in ihrer prototyischen, d.h. ursprünglichen und nicht technisch oder elektronisch vermittelten Form, in der folgenden Weise benennen und ordnen" (Quasthoff 1996, 15). 
Formen (für den Bereich der gesprochenen Sprache etwa das Selbstgespräch oder besagtes Besprechen von Anrufbeantwortern, für geschriebene Sprache beispielsweise das Schreiben von Notizzetteln oder das Ausfüllen von Formularen) werden dabei ausgeblendet - mit dem Effekt einer künstlichen, nicht gegenstandsangemessenen Homogenisierung von Mündlichkeit und Schriftlichkeit.

Ein zweiter Beitrag zur Vereinheitlichung ergibt sich aus der Homogenisierung, die der Vergleichsprozedur inhärent ist: Die Kategorien ,Mündlichkeit' und ,Schriftlichkeit' bzw. ,gesprochene Sprache' und ,geschriebene Sprache' suggerieren einerseits, daß zwischen ihnen eine deutliche Differenz besteht, und andererseits, daß das, was sie bezeichnen, jeweils für sich eine gewisse Homogenität besitzt. Die begriffliche Gegenüberstellung wirkt intern jeweils homogenisierend und zugleich extern differenzenverstärkend. Interne Homogenität ist aber - wie gesagt - nicht gegeben, und auch die Annahme, daß die Praktiken innerhalb der beiden Gruppen - aufgrund ihrer Mündlichkeit bzw. Schriftlichkeit - jeweils mehr mitcinander zu tun haben als mit denen der anderen Gruppe, trifft nicht zu. Auch wenn die Beichte und die Neujahrsansprache des Bundespräsidenten beides mündliche Praktiken sind, scheint es doch evident, daß sie weitaus weniger Gemeinsamkeiten haben als der mündliche mit dem schriftlichen Klatsch.

Einen dritten Beitrag zur Vereinheitlichung leistet die Abstraktion von der Praktikengebundenbeit des Sprechens: In dem Maße, wie Mündlichkeit und Schriftlichkeit als homogene Bereiche konstituiert werden, kann von der Praktikengebundenheit des Sprechens und Schreibens abstrahiert werden. Sprechen und Schreiben erscheinen dann als verallgemeinerte praktikenunabhängige Tätigkeiten, die - wo immer sie auch vorkommen - den gleichen Bedingungen unterliegen und die gleichen Eigenschaften besitzen. Mit der Unterscheidung von Sprechen und Schreiben (unabhängig von den Praktiken, in denen diese Tätigkeiten erscheinen) ist dann auch die Konstruktion eines grundlegenden, polaren Gegensatzes vollzogen. In dem $\mathrm{Maßc}$, wie die Praktikengebundenheit aus dem Blickfeld gerät, tritt auch in den Hintergrund, daß es für Mündlichkeit bzw. Schriftlichkeit spezifische Domänen gibt, und sie erscheinen als ubiquitär konkurrierend.

Resultat dieser drei Prozesse ist die Konstitution von homogen erscheinenden Gegenstandsbereichen gesprochene und geschriebene Sprache.

Im folgenden soll kurz veranschaulicht werden, wie in konkreten Arbeiten der Gesprochenen-Sprache-Forschung mit dem Problem der Vielfalt mündlicher kommunikativer Praktiken umgegangen wird und wie sich die eben beschriebenen Prozesse dort niederschlagen.

Im Grundsatz konkurrieren bei der Beschäftigung mit gesprochener Sprache zwei Sichtweisen: eine medial-extensionale und eine ideal- oder prototypisch-normative. Die medial-extensionale Sichtweise versteht alle die Formen als Mündlichkeit, bei denen Verständigung in irgendeiner Weise mittels Sprechen erfolgt. Die Medialität ist das alleinige Kriterium dafür, was zur Mündlichkeit gerechnet wird. Beim prototypisch-normativen Zugang spielen zusätzliche Kriterien eine Rolle. Den verschiedenen Formen medial-mündlicher Verständigung wird dabei eine Gewichtung aufgeprägt: bestimmte Formen sind deutlicher, besser oder klarer mündlich als andere. Dies beinhaltet die Möglichkeit, ,schlechtere' Fälle aus der Betrachtung oder - weitergehend - als keine, echten' Fälle von Mündlichkeit aus dem Gegenstandsbereich auszuschließen. 
Sowohl das Freiburger Projekt, Grundstrukturen' wie auch Koch und Oesterrcicher, die der Gesprochenen-Sprache-Forschung in der Bundesrepublik wesentliche Impulse gegeben haben, sind - auf unterschiedliche Weise - einer prototypischnormativen Sichtweise verpflichtet.

Im Freiburger Projekt wird durch Ausgrenzungen, wie sie in folgender Definition von Steger deutlich werden, so etwas wie prototypische Mündlichkeit inthronisicrt:

„Als gesprochene Sprache kann [...] nur akzeptiert werden [sic!]

1. was gesprochen wird, ohne vorher aufgezeichnet worden zu sein;

2. was gesprochen wird, ohne länger für einen bestimmten Vortragszweck bedacht worden zu sein.

Schließlich sollte praktischerweise nur Sprache akzeptiert werden, die

3. gesprochen wird, ohne in Vers, Reim oder vergleichbar fester Bindung zu stehen; auch wenn es sich um immer unschriftliche Formen und Formeln handelt.

Das heißt, es scheint gegenwärtig nützlich, nur gesprochene Sprache zuzulassen, dic mit Prosamustern kodiert wird." (Steger 1967, 262)

„Es darf [...] wohl nur akzeptiert werden, was

4. gesprochen wird und im Rahmen des jeweils gesprochenen Sprachtyps als [...] richtig anzuschen ist." (Steger 1967, 264)

Nicht angesprochen ist in dieser Bestimmung die Beschränkung auf Standardsprache, dic gerade für dic Korpuszusammenstellung des Freiburger Projekts konstitutiv ist. Der Zweck dieser Ausgrenzung ist klar: Man möchte, da man mit Schriftlichkeit vergleichen will, nur die Formen der Mündlichkeit berücksichtigen, die möglichst schriftfern sind. Die Korpuserstellung erfolgt dann auf der Grundlage dieses eingeschränkten Verständnisses von Mündlichkeit. Auf diese Weise wird ein partikuläres und einscitiges Bild von Mündlichkeit erzeugt - zunächst nur im Projekt, über dic Wirkung der Korpora aber dann auch im Bewußtsein vieler Linguisten.

Auch Koch/Oesterreicher $(1985,1994)$ etablieren eine ideal- bzw. prototypische Struktur, wenn sic im konzeptionellen Bereich ein Kontinuum der Kommunikationsformen zwischen den Polen ,konzeptionell mündlich' und ,konzeptionell schriftlich' annehmen $(1985,21)$ und indem sic von extremer Mündlichkeit bzw. Schriftlichkeit $(1985,19)$ sprechen. Je näher Kommunikationsformen am Pol konzeptioneller Mündlichkeit liegen, desto ,klarcre', ,dcutlichere' Fälle sind es. Auch wenn dieses Vorgehen nicht ausgrenzend ist, etabliert es doch Prototypik durch die Anordnung relativ zu den Kriterien Nähe und Distanz. Grundlage und Voraussetzung für diese Anordnung sind Vorstellungen darüber, was prototypische Mündlichkeit und Schriftlichkeit (bzw. Nähe und Distanz) ausmacht und wie nahe sich cinzelne Kommunikationsformen jeweils daran befinden.

Eine andere Form des Umgangs mit der Vielfalt des Mündlichen kann - excmplarisch für viele andere Arbeiten - an dem Aufsatz Chafe/Danielewicz (1987) verdeutlicht werden. Der Titel der Untersuchung lautet: Properties of Spoken and Written Language, spricht also von gesprochener und geschriebener Sprache im allgemeinen. Was aber de facto empirisch untersucht wird, sind dann ganz konkret ,conversations' und ,lectures' als mündliche kommunikative Praktiken und, letters' und ,academic papers' als schriftliche. Dadurch, daß auf bestimmte Praktiken rekurriert wird (und werden muß), bestehen im Grundsatz zwei Gefahren: Entweder daß die untersuchten Praktiken als prototypisch angesehen werden und dadurch andersartige Eigenschaften von anderen Praktiken ausgeblendet werden, oder daß die an partikulärem Material gewonnenen Ergebnisse übergeneralisiert werden, in dem 
Sinne, daß die an einer oder an wenigen Praktiken gefundenen Merkmale als für gesprochene Sprache generell geltend ausgegeben werden.

Das zentrale Problem für all diese Versuche, Mündlichkeit und Schriftlichkeit zu kontrastieren, ist die Vielfältigkeit und Inhomogenität sowohl mündlicher wie schriftlicher Kommunikation. Während Koch/Oesterreicher damit immerhin anordnend, also systematisierend, umgehen, wird dic Viclfalt und Hetcrogenität in vielen anderen Fällen beschnitten oder eskamotiert.

\subsection{Das Konzept der kommunikativen Praktiken}

Wir sprechen und schreiben nicht schlechthin, sondern jedes Sprechen und Schreiben geschieht in und ist Bestandteil von kommunikativen Praktiken. Wir sprechen im Rahmen eines Kaffeeklatsches, einer Dienstbesprechung, ciner tclefonischen Vereinbarung cines Arzttermins, einer Rede, einer Theaterrolle etc.; wir schrciben cinen Brief, einen Aufsatz, ein Protokoll, einen Einkaufszettel etc. Jede Verständigung besteht in der Realisierung eines konkreten, singulären Exemplars einer solchen kommunikativen Praktik. Wir verständigen uns nicht, frei', sondern immer nur im Rahmen der uns verfügbaren kommunikativen Praktiken, indem wir ein Exemplar einer solchen Praktik intendieren und realisieren (und dadurch die Praktik zugleich auch fortschreiben und weiterentwickeln).

Alltagsweltlich wird ein sehr breites Spektrum solcher Praktiken unterschieden, und zum großen Teil gibt es für die unterschiedenen Formen auch spezifische Benennungen. SprecherInnen verfügen über ein Wissen, welche Praktiken es in einer Gesellschaft gibt und welche sie aktiv oder passiv beherrschen. Kommunikative Praktiken sind also zunächst ein Konzept der Beteiligten, an dem sic sich orientieren und mit dessen Hilfe sie ihre kommunikative Praxis - produktiv wie rezeptiv - strukturieren und organisieren. Wissenschaftlich werden diese kommunikativen Praktiken als verschiedene Diskurstypen oder Textsorten bzw. als kommunikative Gattungen etc. thematisiert und rekonstruiert.

Kommunikative Praktiken sind zugleich soziale Praktiken, Formen sozialer Praxis. Es handelt sich um gesellschaftlich herausgebildete konventionalisierte Formen zur Bearbeitung rekurrenter kommunikativer $Z$ wecke. Jede Gesellschaft verfügt als Repertoire für die Verständigung über einen spezifischen Satz solcher kommunikativen Praktiken. Als soziale Phänomene sind kommunikative Praktiken geregelt. Das Ausführen einer kommunikativen Praktik bedeutet die Berücksichtigung cines spezifischen Satzes von sozialen Regeln/Konventionen, von denen cin Teil auch sprachlich-kommunikative Konventionen sind. Für jede kommunikativc Praktik ist ein solcher Satz von Regeln konstitutiv, wobei die einzelnen Konventionen durchaus unterschiedlich wichtig sein können. Werden dic Regeln (zumindest dic zentralen) nicht befolgt, so wird nicht die betreffende kommunikative Praktik realisiert; vielleicht jedoch eine andere. Um eine Praktik zu beschreiben, ist es notwendig, die Gesamtheit ihrer Regeln anzugeben.

Das Konzept der kommunikativen Praktiken besitzt Übereinstimmungen, aber auch Unterschiede zu dem der kommunikativen Gattungen (vgl. Luckmann 1988, Bergmann/Luckmann 1995 und Günthner 1995):

"Gattungen bezeichnen also sozial verfestigte und komplexe kommunikative Muster, an denen sich Sprecher/innen und Rezipient/innen sowohl bei der Produktion als auch Interpretation interaktiver Handlungen orientieren." (Günthner 1995, 199) 
„Solche verfestigten Muster, die kommunikative Vorgänge vorzeichnen, indem sie Bestandteile dieser Vorgänge mehr oder minder detailliert und verpflichtend festlegen, werden in der anthropologischen Linguistik und der Wissens- und Sprachsoziologie als ,kommunikative Gattungen' (Luckman 1986) bezeichnet." (Günthner 1995, 193)

Was hier als kommunikative Gattungen angesprochen wird, ist von rigideren und deutlicheren Ordnungsstrukturen geprägt, als es bei kommunikativen Praktiken der Fall ist, was aber nicht besagen soll, daß kommunikative Praktiken keine oder nur unklar ausgeprägte Ordnungsstrukturen besäßen.

Der deutlichste Unterschied besteht in der Einschätzung der Präformiertheit der kommunikativen Praxis durch Praktiken. Das Konzept der kommunikativen Gattungen sicht größere Anteile der kommunikativen Praxis als nicht präformiert und spontan an:

„Many communicative processes are not constrained in the selction and composition of communicative elements in the comparatively rigid form characteristic of a communicative genre. [...]

However, such more or less, spontancous' acts are by no means the only ones to be found among the communicative processes in a society. Probably, they are not even the oncs that occur most frequently. There are others in which the individual follows a recognizable overall model both for selecting elements from the various available communicative codes, espacially language, and for joining them together into units larger than sentences and single messages." (Bergmann/Luckmann 1995, 290)

Das Konzept der kommunikativen Praktiken geht von ciner wcitergehenden Vorstrukturierung aus. Darüber hinaus verdeutlicht der Begriff der Praktik m.E. besser den Vollzugscharakter, die Interaktivität und die Zweckhaftigkeit des kommunikativen Handelns, als der Begriff der Gattung dies tut. ${ }^{18}$

Anders als die Gesprochene-Sprache-Forschung hat sich die Gesprächsforschung von vornherein - wenn auch nicht explizit programmatisch - an solchen kommunikativen Praktiken orientiert. Wenn sie Unterrichtskommunikation, Wegbeschreibungen, Erzählungen, Beratungen, Schlichtungsgespräche etc. als Untersuchungscinheiten wählt und für sie spezifische Handlungsschemata, Muster, Regelungen des Rederechts etc. herausarbeitet, dann rekonstruiert und charakterisiert sic just solche kommunikativen Praktiken und die sie konstituierenden Regeln.

Fragen wir uns abschließend vor dem Hintergrund des Konzepts kommunikativer Praktiken, wo man ,dic' gesprochene Sprache oder ,die' geschriebene Sprache findet: Man findet sie nie allgemein, sondern immer nur in Form von Exemplaren je konkretcr Praktiken. Was wir vorfinden, wenn wir uns empirisch der Wirklichkcit des Sprechens zuwenden, ist nicht gesprochene Sprache schlechthin, sondern es sind einzelne Exemplare konkreter, unterschiedlicher Praktiken.

${ }^{18}$ Zudem crscheint mir der Gattungsbegriff durch literaturwissenschaftliche Gattungskonzeptionen zu stark vorbelastet. 


\section{Literatur}

Altmann, Hans (1981): Formen der „Herausstellung “ im Deutschen. Tübingen: Niemeyer.

Auer, Peter (1991): Vom Ende deutscher Sätze. In: Zeitschrift für Germanistische Linguistik 19, 139-157.

Auer, Peter (1996): The pre-front field position in spoken German and its relvance as a grammaticalization position. In: Pragmatics 6 (3), 295-322.

Auer, Peter (1998): Zwischen Parataxe und Hypotaxe: , abhängige Hauptsätze“ im Gesprochenen und Geschriebenen Deutsch. In: Zeitschrift für Germanistische Linguistik 26, 284-307.

Behaghel, Otto (1899): Geschriebenes Deutsch und gesprochenes Deutsch. Wiederabgedruckt in: Behaghel, Otto (1967): Von deutscher Sprache. Wiesbaden: Sändig, 11-34.

Betten, Anne (1977): Erforschung gesprochener deutscher Standardsprache. Teil 1. In: Deutsche Sprache 5, 335-361.

Bergmann, Jörg/Luckmann, Thomas (1995): Reconstructive genres of everyday communication. In: Aspects of Oral Communication, hrsg. von Uta M. Quasthoff. Berlin: de Gruyter, 289-304.

Chafe, Wallace/Danielewicz, Jane (1987): Properties of Spoken and Written Language. In: Comprehending Oral and Written Language, hrsg. von Rosalind Horowitz und S. Jay Samuels. San Diego etc.: Academic Press, 83-113.

Ehlich, Konrad (1986): Der Normverstoß im Regelwerk. Über den Solözismus. In: Zeitschrift für Literaturwissenschaft und Linguistik 62, 74-91.

Ehlich, Konrad/Switalla, Bernd (1976): Transkriptionssysteme - Eine exemplarische Übersicht. In: Studium Linguistik 2, 78-105.

Fiehler, Reinhard/Barden, Birgit/Elstermann, Mechthild/Kraft, Barbara (i.V.): Aspekte der gesprochenen Sprache.

Ford, Cecillia (1993): Grammar in Interaction. Cambridge: Cambridge University Press.

Gohl, Christine/Günthner, Susanne (1999): Grammatikalisierung von weil als Diskursmarker in der gesprochenen Sprache. In: Zeitschrift für Sprachwissenschaft 18.1, 39-75.

Günther, Hartmut (1995): Die Schrift als Modell der Lautsprache. In: Osnabrücker Beiträge zur Sprachtheorie, H. 51, 15-32.

Günthner, Susanne (1995): Gattungen in der sozialen Praxis: die Analyse ,kommunikativer Gattungen' als Textsorten mündlicher Kommunikation. In: Deutsche Sprache 23, 193-218.

Günthner, Susanne (1999): Entwickelt sich der Konzessivkonnektor obwobl zum Diskursmarker? Grammatikalisierungstendenzen im gesprochenen Deutsch. In: Linguistische Berichte, H. 180, 409-446.

Hoffmann, Ludger (1991): Anakoluth und spracbliches Wissen. In: Deutsche Sprache 19, 97-120.

Hoffmann, Ludger (1996): Satz. In: Deutsche Sprache 24, 193-222.

Hoffmann, Ludger (1998): Parenthesen. In: Linguistische Berichte, H. 175, 299-328.

Hoffmann, Ludger (1999): Ellipse und Analepse. In: Grammatik und mentale Prozesse, hrsg. von Angelika Redder und Jochen Rehbein, Tübingen: Stauffenberg, 69-90.

Kindt, Walther (1994): Satzbegriff und gesprochene Sprache. In: Lingua 94, 25-48. 
Koch, Peter/Oesterreicher, Wulf (1985): Sprache der Nähe - Sprache der Distanz: Mündlichkeit und Scbriftlichkeit im Spannungsfeld von Sprachtheorie und Sprachgeschichte. In: Romanistisches Jahrbuch 36, 15-43.

Koch, Pcter/Oesterreicher, Wulf (1994): Schriftlichkeit und Sprache. In: Schrift und Schriftlichkeit. Ein interdisziplinäres Handbuch internationaler Forschung, hrsg. von Hartmut Günther und Otto Ludwig. Berlin/New York: de Gruyter, 1. Halbband, 587-604.

Krämer, Sybillc (1996): Sprache und Schrift oder: Ist Scbrift verschriftcte Sprache? In: Zeitschrift für Sprachwissenschaft 15.1, 92-112.

Linell, $\operatorname{Per}$ (1982): The written language bias in linguistics. Linköping: University of Linköping. (= Studies in Communication 2).

Luckmann, Thomas (1988): Kommunikative Gattungen im kommunikativen ,Haushalt einer Gesellschaft. In: Der Ursprung der Literatur, hrsg. von G. Smolka-Koerdt, P. M .Spangenberg, D. Tillmann-Bartylla. München, 279-288.

Ludwig, Otto (1980): Geschriebene Sprache. In: Lexikon der Germanistischen Linguistik, hrsg. von Hans Peter Althaus u.a. Tübingen: Nicmeyer, 323-328. 2. Aufl. Lyons, John (1983): Die Sprache. München: Beck.

Motsch, Wolfgang (1992): Ist die Sprechakttheorie eine Theorie der gesprochenen Spracbe? In: Schreibprozesse - Schreibprodukte. Festschrift für Gisbert Keseling, hrsg. von Manfred Kohrt und Arne Wrobel. Hildesheim/Zürich/New York: Olms, 243-253.

Ochs, Elinor/Schegloff, Emanuel A./Thompson, Sandra A. (1996): Interaction and grammar. Cambridge: Cambridge University Press.

Olson, David (1993): How writing represents speech. In: Language \& Communication 13, 1-17.

Quasthoff, Uta M. (1996): Mündliche Kommunikation als körperliche Kommunikation: Beobachtungen zur direkten Interaktion und zum Fernseben. In: Mündlichkeit und Schriftlichkeit im Fernsehen, hrsg. von Bernd Ulrich Biere und Rudolf Hoberg. Tübingen: Narr, 9-28.

Rchbcin, Jochen (1979): Sprechhandlungsaugmente: zur Organisation der Hörersteuerung. In: Die Partikeln der deutschen Sprache, hrsg. von Harald Weydt. Berlin usw.: de Gruyter, 58-74.

de Saussurc, Ferdinand (1967): Grundfragen der allgemeinen Spracbwissenschaft. 2. Auflage. Berlin: de Gruyter.

Schegloff, Emanucl A./Ochs, Elinor/Thompson, Sandra A. (1996): Introduction. In: Interaction and grammar, hrsg. von Elinor Ochs, Emanuel A. Schegloff, Sandra A. Thompson. Cambridge: Cambridge University Press, 1-51.

Scheutz, Hannes (1997): Satzinitiale Voranstellung im gesprochenen Deutsch als Mittel der Themensteuerung und Referenzkonstitution. In: Syntax des gesprochenen Deutsch, hrsg. von Peter Schlobinski. Opladen: Westdeutscher Verlag, 27-54.

Schröder, Peter (1998): Zur Reinterpretation traditioneller grammatischer Kategorien im Rabmen interaktionsorientierter Grammatikkonzepte: "Anakoluthe" mit ikonischer Qualität. In: Sprachgermanistik in Skandinavien III. Akten des IV. Nordischen Germanistentreffens auf Schloß Snadbjerg vom 5.-8. Juni 1996, 57-80.

Selting, Margret (1993): Voranstellungen vor den Satz. Zur grammatischen Form und interaktiven Funktion von Linksversetzung und Freiem Thema im Deutschen. In: Zeitschrift für Germanistische Linguistik 21, 291-319. 
Selting, Margret (1994): Konstruktionen am Satzrand als interaktive Ressource. In: Was determiniert Wortstellungsvariation? Studien zu einem Interaktionsfeld von Grammatik, Pragmatik und Sprachtypologie, hrsg. von Brigitte Haftka. Opladen: Westdeutscher Verlag, 299-318.

Selting, Margret (1995): Der, mögliche Satz' als interaktiv relevante syntaktische Kategorie. In: Linguistische Berichte, H. 158, 298- 325.

Selting, Margret (1997): Sogenannte, Ellipsen' als interaktiv relevante Konstruktionen? Ein neuer Versuch über die Reichweite und Grenzen des Ellipsenbegriffs für die Analyse gesprochener Sprache in der konversationellen Interaktion. In: Syntax des gesprochenen Deutsch, hrsg. von Peter Schlobinski. Opladen: Westdeutscher Verlag, 117-155.

Steger, Hugo (1967): Gesprochene Sprache. Zu ibrer Typik und Terminologie. In: Satz und Wort im heutigen Deutsch. Düsseldorf, 259-291. (= Sprache der Gegenwart 1)

Stcger, Hugo (1987): Bilden „gesprochene Sprache ${ }^{\alpha}$ und „geschriebene Sprache ${ }^{\alpha}$ eigene Sprachvarietäten? In: Wörter: Schätze, Fugen und Fächer des Wissens. Festgabe für Theodor Lewandowski zum 60. Geburtstag, hrsg. von Hugo Aust. Tübingen: Narr, 35-58.

Uhmann, Susanne (1993): Das Mittelfeld im Gespräch. In: Wortstellung und Informationsstruktur, hrsg. von Marga Reis. Tübingen: Niemeyer, 313-354.

Vachek, Josef (1976): Geschriebene Sprache. Allgemeine Probleme und Probleme des Englischen. In: Grundlagen der Sprachkultur. Beiträge der Prager Linguistik zur Sprachtheorie und Sprachpflege. Teil 1, hrsg. von Jürgen Scharnorst und Erika Ising. Berlin: Akademie, 240-295. 